\title{
FINITE ABELIAN SURFACE COVERINGS $\dagger$
}

\author{
by S. A. JASSIM
}

(Received 22 March, 1983)

Introduction. Let $G$ be a finite abelian group, and $Y$ be a closed surface. The problems of classifying and enumerating the free and effective $G$-actions on $Y$ modulo selfhomeomorphisms of $Y$ and $X=Y / G$ can be transferred into ones of classifying regular $G$-coverings on $X$. P. A. Smith [7], proved that for any prime number $p$ there are $p^{r(r-1) / 2}$ equivalence classes of free $\left(\mathbb{Z}_{\mathrm{p}}\right)^{r}$ actions on $Y$ provided that $r \leqq$ genus of $X$. This paper is devoted to the classification and the enumeration of regular $G$-covering surfaces, when $G$ is any finite abelian group. Recently, A. Edmonds [2] classified the $G$-actions on closed surfaces by their $G$-bordism classes in the set $\Theta_{2}^{\text {free }}(G)$ of free oriented $G$-cobordism classes of free oriented $G$-surfaces.

Section 1 is introductory. In Section 2 the classification problems for a finite abelian group $G$ are reduced to the corresponding, reasonably easier, problems for its primary components. In Section 3 Smith's result is generalized for arbitrary $p$-groups. The last section is aimed at the enumeration of weak equivalence classes of regular finite abelian surface coverings. Throughout all coverings are regular, all surfaces are oriented and all surface maps are assumed to be orientation preserving. $H_{*}(-)$ stands for homology with integral coefficients.

1. Let $G$ be a group, $X$ be a closed surface of genus $g$, and $q: Y \rightarrow X$ be a $G$-covering. Then $G$ acts freely on $Y$, and if $G$ is finite then $Y$ is also a closed surface of genus $g^{\prime}=|G|(g-1)+1$. On the other hand, if $G$ acts freely on a space $Y$ then the natural projection $Y \rightarrow Y / G$ is a $G$-covering. Hence there is a bijection between the class $\operatorname{Cov}(X, G)$ of $G$-coverings of $X$ and the set of free $G$-actions on closed surfaces of genus $g^{\prime}$. Moreover, for any $x$ in $X$ the fibre $q^{-1}(x)$ is a $\pi_{1}(X, x)$-set and there exists an onto map from $\operatorname{Cov}(X, G)$ to the class of all short exact sequences of the form

$$
M \stackrel{i}{\rightarrow} \pi_{1}(X, x) \rightarrow G \quad\left(\pi_{1}(q)\right),
$$

where $M=\pi_{1}(Y, y), i=q_{*}$ and $y$ is in the fibre $q^{-1}(x)$. If $G$ is abelian then the isomorphism classes of $G$-coverings $q: Y \rightarrow X$ are in bijection with the set of short exact sequences of abelian groups of the form

$$
q_{*}\left(H_{1}(Y)\right) \stackrel{\alpha}{\rightarrow} H_{1}(X) \stackrel{\beta}{\rightarrow} G \quad(H(q)) .
$$

Two $G$-coverings $q_{i}: Y_{i} \rightarrow X$, for $i=1,2$, are said to be weak equivalent (or simply w-equivalent) if there exist homeomorphisms $F: Y_{1} \rightarrow Y_{2}$ and $f: X \rightarrow X$ such that $q_{2} F=$ $f q_{1}$. If in addition $F$ is a $G$-map then $q_{1}$ and $q_{2}$ are said to be equivalent.

† The results here are presented in the author's Ph.D. thesis written at the University College of Swansea under the supervision of Dr. Alan Thomas to whom the author is most indebted for his constant help and insights.

Glasgow Math. J. 25 (1984) 207-218. 
1.1 Proposition. Two G-coverings $q_{1}$ and $q_{2}$ over $X$ are $w$-equivalent (respectively equivalent) iff the sequences $\pi_{1}\left(q_{i}\right), i=1,2$, enter in a commutative diagram of the form

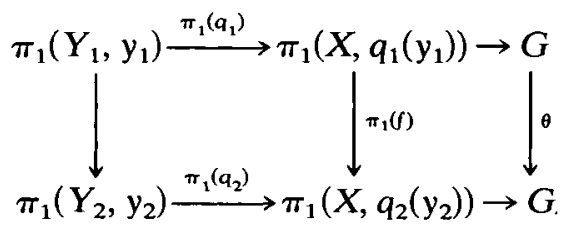

where $f$ is a homeomorphism of $X$ and $\theta$ is an automorphism (resp. an inner automorphism) of $G$.

The proof in the case of w-equivalence follows from the fact that $q_{1}$ and $q_{2}$ are w-equivalent iff $f q_{1}$ and $q_{2}$ are isomorphic for some homeomorphism $f$ of $X$. For the equivalence case see [7].

1.2 Corollary. If $G$ is abelian then two $G$-coverings $q_{1}$ and $q_{2}$ over $X$ are wequivalent (resp. equivalent) iff there exists a commutative diagram of the form

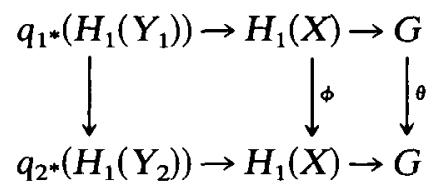

such that $\phi$ is induced by a homeomorphism of $X$ and $\theta$ is an automorphism (resp. the identity map) of $G$.

In certain cases homology with coefficients other than integers may be used. For details see [7] and [4]. The integral first homology group $H_{1}(X)$ is isomorphic to $\mathbb{Z}^{28}$ and the intersection product defines a symplectic form $\langle$,$\rangle on H_{1}(X)$. Throughout a symplectic basis $\left\{a_{1}, \ldots, a_{\mathrm{g}}, b_{1}, \ldots, b_{\mathrm{g}}\right\}$ for $H_{1}(X)$ is fixed, i.e.

$$
\left\langle a_{i}, b_{i}\right\rangle=-\left\langle b_{i}, a_{i}\right\rangle=1 \text { for } i=1, \ldots, g
$$

and if $i \neq j$ then

$$
\left\langle a_{i}, b_{j}\right\rangle=\left\langle a_{i}, a_{i}\right\rangle=\left\langle b_{j}, b_{i}\right\rangle=0 .
$$

It is well known (see [6]) that the group $H^{+}(X)$ of all automorphisms of $H_{1}(X)$ which are induced by orientation preserving homeomorphisms of $X$ is the group of symplectic automorphisms of $H_{1}(X)$. That is, $H^{+}(X)$ is isomorphic to $\mathrm{Sp}_{28}(\mathbb{Z})$. Therefore in 1.2 the statement " $\phi$ is induced by a homeomorphism of $X$ " can be replaced by " $\phi$ is a symplectic automorphism of $H_{1}(X)$ ".

2. For any natural number $g$ and any group $G$, let $W E(g, G)$ and $E(g, G)$ be the sets of w-equivalence and equivalence classes, respectively, of $G$-coverings of the closed surface $X$ of genus $g$. In this section, we obtain, for a direct decomposition $G=\bigoplus_{i=1}^{k} G_{i}$, a 
relation between the cardinality of $W E(g, G)$ (resp. $E(g, G))$ and those of the $W E\left(g, G_{i}\right)$ 's (resp. $E\left(g, G_{i}\right)$ 's).

Throughout this section $H=H_{1}(X), G_{1}$ and $G_{2}$ are finite abelian groups of relatively prime orders, $n_{i}: G_{i} \rightarrow G_{1} \oplus G_{2}$ is the natural injection, for $i=1,2$, and for any group $G$, $|H, G|$ stands for the set of epimorphisms from $H$ onto $G$. Two elements in $|H, G|$ are said to be w-equivalent (resp. equivalent) if they correspond to w-equivalent (resp. equivalent) $G$-coverings of $X$. Let $\Psi$ be the isomorphism

$$
\operatorname{Hom}\left(H, G_{1}\right) \times \operatorname{Hom}\left(H, G_{2}\right) \rightarrow \operatorname{Hom}\left(H, G_{1} \oplus G_{2}\right):\left(\alpha_{1}, \alpha_{2}\right) \rightarrow n_{1} \alpha_{1} \oplus n_{2} \alpha_{2} .
$$

The proof of the following lemma is straightforward. However, it is not necessarily true if $G_{1}$ and $G_{2}$ are not of relatively prime orders.

\subsection{LeMmA. The restriction of $\Psi$ to $\left|H, G_{1}\right| \times\left|H, G_{2}\right|$ defines a bijection}

$$
\psi_{e}:\left|H, G_{1}\right| \times\left|H, G_{2}\right| \rightarrow\left|H, G_{1} \oplus G_{2}\right| .
$$

For the special fixed basis $\left\{a_{1}, \ldots, a_{g}, b_{1}, \ldots, b_{g}\right\}$ of $H$, define:

$$
x_{i}=\left\{\begin{array}{lll}
a_{i} & \text { if } & 1 \leqq i \leqq g, \\
b_{i} & \text { if } & g<i \leqq 2 g
\end{array}\right.
$$

An automorphism $\phi$ of $H$ is said to be quasi-elementary if for each $i=1, \ldots, 2 g$, $\phi\left(x_{i}\right) \neq x_{i}$ implies that there exists a $j \neq i$ such that $\phi\left(x_{i}\right)=x_{i} \mp x_{j}$ and $\phi\left(x_{j}\right)=x_{j}$.

Consider the integral $2 \mathrm{~g} \times 2 \mathrm{~g}$ matrices:

$$
\begin{array}{ll}
\alpha_{i}=\left(\begin{array}{l|l}
I_{\mathrm{g}} & Y_{i} \\
\hline 0 & I_{\mathrm{g}}
\end{array}\right), \quad \beta_{\mathrm{i}}=\alpha_{i}^{\mathrm{i}} & \text { for } i=1, \ldots, g, \\
\lambda_{i}=\left(\begin{array}{c|c}
I_{\mathrm{g}} & 0 \\
\hline-U_{i, i+1} & I_{\mathrm{g}}
\end{array}\right), \quad \sigma_{i}=\left(\begin{array}{l|l}
I_{\mathrm{g}} & T_{i} \\
\hline 0 & I_{\mathrm{g}}
\end{array}\right) \quad \text { for } i=1, \ldots, g-1, \\
\gamma_{\mathrm{ij}}=\left(\begin{array}{c|c}
I_{\mathrm{g}} & 0 \\
\hline-U_{i j} & I_{\mathrm{g}}
\end{array}\right), \quad \gamma_{j i}=\gamma_{i j}^{\mathrm{t}} & \text { for } 1 \leqq i \leqq j \leqq g, \\
\delta_{i j}=\left(\begin{array}{l|l}
Z_{i j} & 0 \\
\hline 0 & \left(Z_{i j}^{-1}\right)^{2}
\end{array}\right), \quad \text { and } \quad \delta_{j i}=\delta_{i j}^{t} & \text { for } 1 \leqq i \leqq j \leqq g,
\end{array}
$$

where: $Y_{i}$ is the $g \times g$ matrix whose only nonzero entry is $y_{i i}=1$,

$U_{i j}$ is the $g \times g$ matrix whose only nonzero entries are $u_{i j}=u_{j i}=1$,

$Z_{i j}$ is the $g \times g$ matrix obtained from $I_{\mathrm{g}}$ by adding the $i$-th row to the $j$-th row,

$T_{i}$ is the $g \times g$ matrix whose only nonzero entries are $t_{i i}=t_{i+1, i+1}=-1$ and $t_{i, i+1}=t_{i+1, i}=1$.

Birman [1] has shown that $\operatorname{Sp}_{2 \mathrm{~g}}(\mathbb{Z})$ is generated by the set

$$
\left\{\alpha_{i}, \beta_{i}, \sigma_{j} \mid i=1, \ldots, g \text { and } j=1, \ldots, g-1\right\} \text {. }
$$

The relation

$$
\sigma_{i}=\alpha_{i+1}^{-1} \beta_{i}^{-1} \alpha_{i} \alpha_{i+1} \beta_{i+1}^{-1} \alpha_{i+1} \lambda_{i}^{-1} \alpha_{i}^{-1} \beta_{i} \alpha_{i}^{-1} \alpha_{i+1}^{-1} \beta_{i+1} \alpha_{i+1}^{-1}
$$


shows that $\left\{\alpha_{i}, \beta_{i}, \lambda_{j} \mid i=1, \ldots, g\right.$ and $\left.j=1, \ldots, g-1\right\}$ also generates $\mathrm{Sp}_{2 \mathrm{~g}}(\mathbb{Z})$. The last set consists of only quasi-elementary automorphisms of $H$.

2.2 Propostrion. For $i=1,2$ let $h_{i}$ and $k_{i}$ be in $\left|H, G_{i}\right|$. Then $\psi_{e}\left(h_{1}, h_{2}\right)$ is $w$ equivalent (resp. equivalent) to $\psi_{e}\left(k_{1}, k_{2}\right)$ iff $h_{i}$ is w-equivalent (resp. equivalent) to $k_{i}$.

We will only prove the proposition in the case of $w$-equivalence. The other case is similar.

Proof. The necessity is obvious.

To prove the sufficiency, let $\theta_{1}$ be an automorphism of $G_{1}$ and $\phi$ be in $\operatorname{Sp}_{2 g}(\mathbb{Z})$ such that $\theta_{1} h_{1}=k_{1} \phi$. By induction on the number of quasi-elementary factors of $\phi$ we will prove that $\psi_{e}\left(h_{1}, h_{2}\right)$ is w-equivalent to $\psi_{e}\left(k_{1}, h_{2}\right)$.

If $\phi$ is quasi-elementary, then choose positive integers $r_{1}$ and $r_{2}$ such that $r_{1}\left|G_{1}\right|+$ $r_{2}\left|G_{2}\right|=1$, and let $n=r_{1}\left|G_{1}\right|$ and $m=r_{2}\left|G_{2}\right|$. Consider the symplectic automorphism $\phi^{m}$ of $H$ and the automorphism $\theta=\theta_{1} \oplus I_{G_{2}}$ of $G_{1} \oplus G_{2}$. For each $i$, if $\phi\left(x_{i}\right)=x_{i}$, then

$$
\begin{aligned}
\psi_{e}\left(k_{1}, h_{2}\right) \phi^{m}\left(x_{i}\right) & =\psi_{e}\left(k_{1}, h_{2}\right)\left(x_{i}\right)=k_{1}\left(x_{i}\right) \oplus h_{2}\left(x_{i}\right) \\
& =k_{1}\left(\phi\left(x_{i}\right)\right) \oplus h_{2}\left(x_{i}\right)=\left(\theta_{1} h_{1} \oplus I_{G_{2}} h_{2}\right)\left(x_{i}\right) \\
& =\theta \psi_{e}\left(h_{1}, h_{2}\right)\left(x_{i}\right) .
\end{aligned}
$$

But if $\phi\left(x_{i}\right) \neq x_{i}$ then there exists a $j$ such that $\phi\left(x_{i}\right)=x_{i} \mp x_{j}$ and $\phi\left(x_{j}\right)=x_{j}$. In this case $\phi^{m}\left(x_{i}\right)=x_{i} \mp m x_{j}$ and

$$
\begin{aligned}
\psi_{e}\left(k_{1}, h_{2}\right) \phi^{m}\left(x_{i}\right) & =k_{1}\left(x_{i} \mp m x_{i}\right) \oplus h_{2}\left(x_{i} \mp m x_{i}\right) \\
& =\left(k_{1}\left(x_{i} \mp x_{j}\right) \pm n k_{1}\left(x_{j}\right) \oplus\left(h_{2}\left(x_{i}\right) \mp m h_{2}\left(x_{j}\right)\right)\right. \\
& =k_{1} \phi\left(x_{i}\right) \oplus h_{2}\left(x_{i}\right)=\left(\theta_{i} h_{1} \oplus I_{G_{2}} h_{2}\right)\left(x_{i}\right)=\theta \psi_{e}\left(h_{1}, h_{2}\right)\left(x_{i}\right) .
\end{aligned}
$$

Consequently $\psi_{e}\left(h_{1}, h_{2}\right)$ is w-equivalent to $\psi_{e}\left(k_{1}, h_{2}\right)$.

If $\phi=\phi_{1} \phi_{2} \ldots \phi_{r}$ for some quasi-elementary automorphisms $\phi_{1}, \ldots, \phi_{r}$ of $H$, then $k_{1} \phi_{1}$ is w-equivalent to $k_{1}$. By the first case $\psi_{e}\left(k_{1} \phi_{1}, h_{2}\right)$ is w-equivalent to $\psi_{e}\left(k_{1}, h_{2}\right)$. By the induction hypothesis, $\psi_{e}\left(k_{1} \phi_{1}, h_{2}\right)$ is w-equivalent to $\psi_{e}\left(h_{1}, h_{2}\right)$. By transitivity, then, $\psi_{e}\left(k_{1}, h_{2}\right)$ is w-equivalent to $\psi_{e}\left(k_{1}, h_{2}\right)$.

Similarly, $\psi_{e}\left(k_{1}, h_{2}\right)$ is w-equivalent to $\psi_{e}\left(k_{1}, k_{2}\right)$, and the proposition follows by transitivity.

2.3 COROLlary. The function $\psi_{e}$ induces bijections

$$
E\left(\mathrm{~g}, G_{1}\right) \times E\left(\mathrm{~g}, G_{2}\right) \rightarrow E\left(\mathrm{~g}, G_{1} \oplus G_{2}\right)
$$

and

$$
\mathrm{WE}\left(\mathrm{g}, \mathrm{G}_{1}\right) \times W E\left(\mathrm{~g}, \mathrm{G}_{2}\right) \rightarrow \mathrm{WE}\left(\mathrm{g}, \mathrm{G}_{1} \oplus \mathrm{G}_{2}\right) .
$$

By induction on the number of $p$-primary subgroups of an abelian group $G$, the above corollary yields the following main object of the present paper.

2.4 Proposition. Let $G$ be a finite abelian group with the p-primary decomposition 
$G=\bigoplus_{i=1}^{r} G_{p_{i}}$, where $p_{1}, \ldots, p_{r}$ are the distinct prime divisors of $G$. Then

$$
\operatorname{Card}(E(g, G))=\prod_{i=1}^{r} \operatorname{Card}\left(E\left(g, G_{i}\right)\right)
$$

and

$$
\operatorname{Card}(W E(g, G))=\prod_{i=1}^{r} \operatorname{Card}\left(W E\left(g, G_{i}\right)\right)
$$

3. Let $p$ be a prime which we will fix throughout the rest of this paper. Let $m_{1}, \ldots, m_{r}$ be positive integral powers of $p$, ordered increasingly, and let $G=\bigoplus_{i=1}^{r} \mathbb{Z}_{m_{i}}$. Let $\left\{g_{1}, \ldots, g_{r}\right\}$ be the basis for $G$ formed by the $2 g$-tuples $g_{i}=\left(g_{i 1}, \ldots, g_{i, 2 g}\right)$, where $g_{i j}=\delta_{i j}$ the kronecker delta. In what follows the product $\prod_{i=1}^{k} T_{i}$ of matrices stands for $T_{1}, T_{2} \ldots T_{k}$, and $\mathbb{Z}_{m}$ is represented additively by $\{0,1, \ldots, m-1\}$.

For any $G$-covering $q: Y \rightarrow X$, let $\beta(q)$ be the $r \times 2 g$ integral matrix that represents the epimorphism $\beta$ in the short exact sequence $H(q)$, given in Section 1 , with respect to the fixed bases for $G$ and $H_{1}(X)$. Note that, here $H(q)$ has the form

$$
\operatorname{ker}(\beta) \rightarrow \mathbb{Z}^{2 \mathrm{R}} \stackrel{\beta}{\rightarrow} G
$$

where $\operatorname{ker}(\beta)=\mathbb{Z}^{2 \mathrm{~g}}$. If $M(\mathrm{~g}, G)$ is the set of all $r \times 2 \mathrm{~g}$ integral matrices of row rank $r$ and whose $i$ th row, $i=1, \ldots, r$, consists of integers in $\mathbb{Z}_{m_{i}}$, then $\beta()$ defines a surjection $\operatorname{Cov}(\mathrm{g}, G) \rightarrow M(\mathrm{~g}, G)$.

Two matrices $A, B$ in $M(\mathrm{~g}, G)$ are said to be equivalent if there exists $R$ in $\operatorname{Sp}_{2 \mathrm{~g}}(\mathbb{Z})$ such that $A=B R$. In this case we write $A \sim B$. By Corollary 1.2 the function $\beta()$ induces a bijection

$$
E(g, G) \rightarrow M(g, G) / \sim,
$$

which is independent of the choice of the special basis for $H$. If a different basis is chosen for $G$ then the corresponding set of matrices is in bijection with $M(g, G)$.

3.1 Definition. Let $A$ be a matrix in $M(g, G)$. For any two integers $i$ and $j$ with $1 \leqq i \leqq j \leqq r$, define

$$
\langle A\rangle_{i j}=\left\langle r_{i}, r_{j}\right\rangle \bmod m_{i},
$$

where $\left\langle r_{i}, r_{j}\right\rangle$ is the symplectic product of the $i$ th and $j$ th rows of $A$. It is easy to prove that if $A$ and $B$ are two equivalent matrices then for each $i$ and $j,\langle A\rangle_{i j}=\langle B\rangle_{i j}$.

3.2 Proposition. If $1 \leqq r \leqq g$ then any matrix $A$ in $M(g, G)$ is equivalent to one of the form $\left(H_{r} \mid U\right)$, where

(1) $H_{r}$ is the $r \times g$ matrix with 1 on its main diagonal and zero elsewhere, and

(2) $U$ is an upper triangular $r \times g$ matrix with zero main diagonal and such that $u_{i j}=0$ for $j>r$. 
This is a generalisation of Proposition (6.1) of Smith's paper [7], and so the proof is omitted.

3.3 COROLlaRy. If $r \leqq g$ then the cardinality of $E(g, G)$ is $p^{\kappa}$, where

$$
K=\sum_{i=1}^{r-1}(r-1) k_{i} \text { and } m_{i}=p^{k_{i}}
$$

This follows from the fact that if $A$ is a matrix in $M(g, G)$ of the form described in Proposition 3.2, then for each $i$ and $j$ with $1 \leqq i \leqq j \leqq r,\langle A\rangle_{i j}=u_{i j}$.

4. In this section the classification of abelian primary coverings of closed surfaces modulo weak equivalence is studied. If $G$ is a finite abelian group then for any $G$-covering $q: Y \rightarrow X$, the short exact sequence $H(q)$ has the form

$$
\operatorname{ker}(\beta) \rightarrow \mathbb{Z}^{2 \mathrm{~g}} \stackrel{\beta}{\rightarrow} G
$$

with respect to the special basis for $H_{1}(X)=\mathbb{Z}^{2 R}$. Since $\operatorname{ker}(\beta) \cong \mathbb{Z}^{2 \mathrm{~g}}$, then for any such covering a basis $\left\{e_{1}, \ldots, e_{2 \mathrm{~g}}\right\}$ can be chosen with respect to which $H(q)$ has the form

$$
\mathbb{Z}^{2 g} \stackrel{\alpha_{a}}{\longrightarrow} \mathbb{Z}^{2 g} \stackrel{\beta}{\rightarrow} G
$$

Let $\alpha(q)$ be the $2 g \times 2 g$ integral matrix that represents the monomorphism with respect to the given bases. If

$$
N(g, G)=\{A \mid A \text { is a } 2 g \times 2 g \text { integral matrix and Coker } A=G\}
$$

then

is a surjection.

$$
\alpha(): \operatorname{Cov}(g, G) \rightarrow N(g, G): q \rightarrow \alpha(q)
$$

Two matrices $A$ and $B$ in $N(g, G)$ are said to be w-equivalent, in symbols $A \approx B$, if $B=L A R$ for some $L$ in $\operatorname{Sp}_{2 \mathrm{~g}}(\mathbb{Z})$ and $R$ in $G L_{2 \mathrm{~g}}(\mathbb{Z})$. By 1.2, the function $\alpha()$ induces a bijection

$$
W E(g, G) \rightarrow N(g, G) / \approx,
$$

which is independent of the different choices of the bases.

4.1 Definition. For any matrix $A$ in $N(g, G)$ and any vector $v$ in $\mathbb{Z}^{2 \mathrm{~g}}$ we define $J_{v}(A)$ to be the principal ideal in $\mathbb{Z}$ generated by the set of inner products $\left\langle c_{1}, v\right\rangle, \ldots$, $\left\langle c_{2 g}, v\right\rangle$, where $c_{i}$ is the $i$ th column of $A$.

If $\langle A\rangle$ is the subgroup of $\mathbb{Z}^{2 \mathrm{~g}}$ generated by the columns of $A$, then $J_{v}(A)$ is generated by $\mid \min \{\langle a, v\rangle \mid a$ in $A\} \mid$. Therefore if $M$ is any matrix in $G L_{2 g}(\mathbb{Z})$ then $J_{v}(A)=J_{v}(A M)$. Moreover, if $A \approx B$ then there exists $S$ in $\operatorname{Sp}_{2 \mathrm{~g}}(\mathbb{Z})$ such that for any $v$ in $\mathbb{Z}^{2 \mathrm{~g}}, J_{v}(A)=$ $J_{\text {Sv }}(B)$.

The following lemma provides a simple, but frequently needed, characterization of the matrices in $\mathrm{Sp}_{2 \mathrm{~g}}(\mathbb{Z})$. For any $2 \mathrm{~g} \times 2 \mathrm{~g}$ matrix $A$ and any set $i_{1}, i_{2}, j_{1}, j_{2}$ of natural numbers less than $2 \mathrm{~g}$, let $A\left(i_{1}, i_{2}, j_{1}, j_{2}\right)$ be the determinant of the $2 \times 2$ submatrix of $A$ 
obtained by deleting all rows and columns but the $i_{1}$ th and $i_{2}$ th rows and the $j_{1}$ th and $j_{2}$ th columns.

4.2 LeMMA. Let $S$ be a $2 g \times 2 g$ integral matrix. The following are equivalent.

(1) $S$ is symplectic,

(2) for each $i_{1}$ and $i_{2}$ with $1 \leqq i_{1} \leqq i_{2} \leqq 2 \mathrm{~g}$

$$
\sum_{j=1}^{\mathrm{g}} S\left(i_{1}, i_{2} ; j, j+\mathrm{g}\right)= \begin{cases}1 & \text { if } i_{2}=i_{1}+g, \\ 0 & \text { otherwise, and }\end{cases}
$$

(3) for each $j_{1}$ and $j_{2}$ with $1 \leqq j_{1} \leqq j_{2} \leqq 2 g$

$$
\sum_{i=1}^{\mathrm{g}} S\left(i, i+g ; j_{1}, j_{2}\right)=\left\{\begin{array}{ll}
1 & \text { if } j_{2}=j_{1}+g \\
0 & \text { otherwise }
\end{array} .\right.
$$

Let $p$ be a fixed prime number, $k_{1}, \ldots, k_{r}$ be non-decreasingly ordered natural numbers, and $G=\bigoplus_{i=1}^{r} \mathbb{Z}_{a_{i}}, a_{i}=p^{k_{i}}$. Obviously an integral matrix $A$ is in $N(g, G)$ iff the elementary divisors of $A$ are those of $G$ (i.e. $A$ is similar to the diagonal matrix $\operatorname{diag}\left(1, \ldots, 1, p^{k_{1}}, \ldots, p^{k_{r}}\right)$. For simplicity we introduce the following notations.

4.3 Notations. For any integer $a \neq 0$, define:

$$
\begin{aligned}
p(a) & =\max \left\{r \mid p^{r} \text { divides } a\right\}, \\
{[a] } & =p^{p(a)}, \text { and } \\
(a) & =a /[a] .
\end{aligned}
$$

And let $p(0)=\infty$ and $(0)=0$.

The elementary column operation of adding the $j$ th column to the $i$ th one will be denoted by $C_{i j}$.

For natural numbers $r, s, t, w, i$, and $j$ with $r s+t w=1$ and $1 \leqq i \leqq j \leqq 2 g$,

$$
\left(\begin{array}{cc}
r & -w \\
t & s
\end{array}\right)_{i j}
$$

stands for the $2 \mathrm{~g} \times 2 \mathrm{~g}$ matrix obtained from $I_{2 \mathrm{~g}}$ by replacing the $i i$ th, $i j$ th, $j i$ th, and $j j$ th entries by $r,-w, t$, and $s$ respectively. If $j=i+g$ then this matrix is symplectic in which case it will be written as

$$
\left(\begin{array}{cc}
r & -w \\
t & s
\end{array}\right)_{i}
$$

The sympletic matrix that interchanges the $i$ th row with the $j$ th row and the $(i+g)$ th row with the $(j+g)$ th row will be denoted by $R_{i j}$.

Finally, by $\mathbb{Z}_{1}$ we mean the trivial group.

4.4 Proposttion. Let $A$ be a $2 \mathrm{~g} \times 2 \mathrm{~g}$ integral matrix whose determinant is $p^{n}$ for some 
natural number $n$. Then $A$ is w-equivalent to a matrix of the form

$$
\left(\begin{array}{l|l}
D & 0 \\
\hline V & U
\end{array}\right)
$$

where $D=\operatorname{diag}\left(d_{11}, \ldots, d_{\mathrm{gg}}\right), U$ is an upper triangular $\mathrm{g} \times \mathrm{g}$ matrix and $V$ is an upper triangular matrix with zero diagonal such that:

(1) $d_{11}|\ldots| d_{\mathrm{gg}}\left|u_{\mathrm{gg}}\right| \ldots \mid u_{11}$ and $\sum_{i=1}^{8}\left(p\left(d_{i i}\right)+p\left(u_{i i}\right)\right)=n$,

(2) $\left(d_{i i}\right)=\left(u_{i i}\right)=1, i=1, \ldots, g$,

(3) for each $i<j$ if $u_{i j} \neq 0$ then $p\left(u_{i j}\right) \leqq p\left(u_{i j}\right)<p\left(u_{i i}\right)$,

(4) for each $i<j$ if $v_{i j} \neq 0$ then $p\left(d_{i j}\right) \leqq p\left(v_{i j}\right)<p\left(u_{i i}\right)$.

Matrices in this form are said to be in canonical form.

Proof. The proof is by induction on $g$.

If $g=1$ then the proof follows from the fact that $S p_{2}(\mathbb{Z})=S L_{2}(\mathbb{Z})$ and a slight modification of the matrix equivalent of the structure theorem of finitely generated abelian groups; see [3, Thm. 7.10].

For $\mathrm{g}>1$, row operations of premultiplication by powers of $\alpha_{1}, \beta_{1}, \gamma_{1 j}$, and $\gamma_{j 1}$ as necessary followed by column operations ensure that, up to equivalence, $a_{11} \equiv d_{11}$ is the only non-zero entry in the first row and the first column, and $a_{11}$ divides all the other entries of $A$. Since all elementary row operations on the rows other than the first and the $(g+1)$ th rows can be carried out by means of symplectic matrices which do not affect the first row, then by the matrix equivalent of the structure theorem one can assume that the first $g$ rows of $A$ are of the form $(D \mid 0)$, where $D=\operatorname{diag}\left(d_{11}, \ldots, d_{\mathrm{gg}}\right)$ and $d_{11}|\ldots| d_{\mathrm{gg}}$. Moreover, column operations on the last $g$ columns can be used to reduce the lower right hand corner into an upper triangular $g \times g$ matrix whose first entry divides all the entries in the $(g+1)$ th row of $A$. Now, by the induction hypothesis $A$ is w-equivalent to one which satisfies all the required conditions except possibily that for some $j>1, u_{i j} \neq 0$ and $p\left(u_{j j}\right)>p\left(u_{1 j}\right)$. But in this case, repeating the above process on the matrix $\delta_{1 j}^{-1} A C_{i 1}^{x}$ with $x=d_{i j} / d_{11}$ yields a matrix with $p\left(u_{i j}\right)$ reduced. Repeating the process as much as necessary together with induction completes the proof.

Different matrices in canonical form (i.e. satisfying the conditions of 4.4) may still be w-equivalent. So further reductions of the canonical forms are essential. The rest of the paper is devoted to studying those cases where such reductions are possible. The first such case is the one of homocyclic $p$-groups.

4.5 Propostrion. Let $g \geqq 2$ be an integer, $k$ and $r$ be natural numbers such that $1<r<2 g-1$, and $G=\left(\mathbb{Z}_{p^{k}}\right)^{r}$. Any matrix $A$ in $N(g, G)$ in canonical form is $w$-equivalent to one in which $U$ is a diagonal matrix and the only possibly non-zero entry of $V$ is $v_{n-1, n}=p^{m}$ with $1 \leqq m<k$ and

$$
n= \begin{cases}r & \text { if } r<g, \\ 2 g-r & \text { otherwise. }\end{cases}
$$

Any such matrix is said to be of the form $K(m)$. 
Proof. The proof is by induction on $\mathrm{g}$.

If $g=2$ then $r=2$ and one need only prove that $v_{12}=p^{m}$ for some $m$, with $1 \leqq m<k$. Suppose that $v_{12}=w p^{m}$ for some $w \neq 0,1$ such that $(w, p)=1$. Choose integers $s_{1}, s_{2}, t_{1}$, and $t_{2}$ such that $s_{1} w+s_{2} p^{k-m}=1$ and $s_{1} t_{1}+p^{m} t_{2}=1$. Then

$$
\left(\begin{array}{cc}
t_{1} & t_{2} \\
-p^{m} & s_{1}
\end{array}\right)_{2} \delta_{21} A\left(\begin{array}{cc}
s_{1} & -p^{k-m} \\
s_{2} & w
\end{array}\right)_{23} C_{21} C_{31}^{p^{k-m}} C_{43}^{-s_{1}} C_{34}^{-t_{1}} C_{42}^{-p^{k-m}}\left(\begin{array}{cc}
0 & 1 \\
-1 & 0
\end{array}\right)_{34}
$$

has the desired form.

Suppose that $g>2$. If $r>g$ then conditions (3) and (4) of 4.4 ensure that $U$ is diagonal and the $g$ th column of $V$ is a zero column. Now the induction hypothesis applied to the $2(g-1) \times 2(g-1)$ submatrix of $A$ formed by deleting the $g$ th and $2 g$ th rows and columns yields the required form. If $r<g$ then in the matrix

$$
\left(\prod_{i=1}^{\mathrm{g}} \gamma_{i \mathrm{~g}}^{u_{i \mathrm{~g}}}\right) S\left(\prod_{i=1}^{\mathrm{g}-1} \delta_{i \mathrm{~g}}^{u_{i \mathrm{~g}}}\right) A\left(\prod_{i=1}^{\mathrm{g}-1} C_{i, 2 \mathrm{~g}}^{-u_{i \mathrm{~g}}}\right)\left(\prod_{i=1}^{g-1} C_{i, 2 \mathrm{~g}}^{u_{i \mathrm{~g}}}\right)
$$

the $g$ th column of $V$ is a zero column and $u_{\mathrm{gg}}=1$ is the only non-zero entry in its column, where $S$ is a product of powers of $\gamma_{i j}$ 's needed to restore the canonical form. Again the induction hypothesis gives the required result.

If $r=\mathrm{g}$, then the induction hypothesis implies that $U=\operatorname{diag}\left(p^{k}, \ldots, p^{k}\right)$ and the only possibly non-zero entries of $V$ are the $v_{1 j}$ 's for $j=2, \ldots, g$ and $v_{g-1, g}=p^{m}$ with $0 \leqq m \leqq$ $k$. If $v_{1 j}=0$ for all $j$ then there is nothing to prove. Otherwise, let $h$ be the first integer such that $p\left(v_{1 h}\right)=\min _{i}\left\{p\left(v_{1 j}\right)\right\}$. Replacing $A$ by the matrix

$$
\delta_{h-1,2}^{-1} \delta_{h-1, h}^{-1} \alpha_{h-1}^{-v_{h}^{-1, h}} A C_{h-1, h} C_{h+\mathrm{g}, h+\mathrm{g}-1}^{-1} C_{2, h-1} C_{\mathrm{g}+h-1, \mathrm{~g}+2}^{-1}
$$

if necessary shows that $h=2$. Now similar operations to those used in the case $g=2$ can be used to make $v_{12}=p^{n}$ for some $n$. If $x_{i}=v_{1 j} / v_{12}$ then in

$$
\prod_{i=3}^{g}\left(\delta_{j 2}^{x_{i}} A C_{j 2}^{-x_{i}}\right)
$$

the $v_{i j}$ 's are replaced by 0 for $j>2$. By now only $v_{12}=p^{h}$ and $v_{g-1, g}=p^{m}$ are non-zero entries of $V$. If $g=3$ and $h \geqq m$ then

$$
\delta_{13}^{-p^{h-m}} \gamma_{12}^{p} A C_{13}^{p^{h-m}} C_{64}^{p^{h-m}}
$$

is of the form $K(m)$. But if $g=3$ and $h<m$, then

$$
\gamma_{12}^{p^{m-h}} R_{13} R_{23} R_{12} A R_{12} R_{23} R_{13} C_{12}^{p^{m-h}} C_{45}^{-p^{m-h}}
$$

is of the form $K(\mathrm{~m})$. If $g=4$, then similar operations to those used in the case $g=3$ applied on the matrix $R_{23} A R_{23}$ yield the required form. For $g>4$, the induction hypothesis applied to the $2(g-1) \times 2(g-1)$ submatrix of

$$
R_{12} A\left(\begin{array}{ll}
0 & 1 \\
1 & 0
\end{array}\right)_{23} R_{12}
$$


obtained by deleting the 1 st and the $(g+1)$ th rows and columns yields a matrix of the form $K(m)$ and this completes the proof.

Note that if $r=1,2 g-1$, or $2 g$ then any matrix in $N\left(g, \mathbb{Z}_{p^{k}}^{r}\right)$ is w-equivalent to the only one in canonical form: $\operatorname{diag}\left(1, \ldots, 1, p^{k}\right), \operatorname{diag}\left(1, p^{k}, \ldots, p^{k}\right)$, or $\operatorname{diag}\left(p^{k}, p^{k}, \ldots, p^{k}\right)$. Therefore by proving that for $m \neq h, K(m)$ is not w-equivalent to $K(h)$, the classification of homocyclic surface coverings is complete. Assume, for a contradiction that $K(m)$ is w-equivalent to $K(h)$. There are two cases.

Case 1: $g \leqq r \leqq 2 g-1$. If $K(m)=S K(m) R$ for $S$ in $\operatorname{Sp}_{2 \mathrm{~g}}(\mathbb{Z})$ and $R$ in $G L_{2 \mathrm{~g}}(\mathbb{Z})$, then the symplectic products of the $(n-1)$ th and the $n$th columns of $K(m)$ and $K(h) R$ are equal. This yields the equation

$$
\begin{aligned}
p^{k} \sum_{i=1}^{\mathrm{g}}\left(r_{i, n-1} r_{i+\mathrm{g}, n}-r_{i+\mathrm{g}, n} r_{i n}\right)+p^{2 k} \sum_{i=n+1}^{\mathrm{g}}\left(r_{i, n-1} r_{i+\mathrm{g}, n}-r_{i+\mathrm{g}, n-1} r_{i n}\right) & +p^{h}\left(r_{n-1, n-1} r_{n n}-r_{n-1, n} r_{n, n-1}\right)=p^{m},
\end{aligned}
$$

which has no integral solution. Therefore $K(m)$ is not w-equivalent to $K(h)$.

Case 2: $1<r<g$. In this case let $q_{1}$ and $q_{2}$ be the $G$-coverings that correspond to $K(m)$ and $K(h)$ respectively. Let $\beta_{1}$ and $\beta_{2}$ be the integral matrices that represent the epimorphisms in the short exact sequences $H\left(q_{1}\right)$ and $H\left(q_{2}\right)$ respectively. Then $\beta_{1}=$ $\left(W \mid H_{r}\right)$ and $\beta_{2}=\left(X \mid H_{r}\right)$, where $H_{r}$ is the $r \times g$ matrix $\left(I_{r} \mid 0\right)$ and the only non-zero entries of $W$ and $X$ are $w_{r-1, r}=p^{k-m}$ and $x_{r-1, r}=p^{k-h}$ respectively. If $\beta_{2}=S \beta_{1} T$ for some $S$ in $S p_{2 \mathrm{~g}}(\mathbb{Z})$ and $T$ in $G L_{r}\left(\mathbb{Z}_{p^{k}}\right)$, then the symplectic product of the $(r-1)$ th and $r$ th rows of $\beta_{2}$ must be equal modulo $p^{k}$ to the product of those of $\beta_{1} T$. But this implies that

$$
p^{k-n} \equiv\left(t_{r-1, r} t_{r, r+g}-t_{r, r} t_{r-1, r+g}\right) p^{k-m} \bmod p^{k},
$$

which cannot hold for any $T$ unless $m=n$.

The above arguments, together with the fact that if $r>2 g$, then there is no epimorphism $H_{1}\left(X_{\mathrm{g}}\right) \rightarrow\left(\mathbb{Z}_{\mathrm{p}^{k}}\right)^{r}$ and hence $\operatorname{Cov}(g, G)=\varnothing$, complete the proof of the following proposition.

4.6 Propostrion. Let $g \geqq 2, k$ and $r$ be positive integers. If $G=\left(\mathbb{Z}_{\mathrm{p}^{k}}\right)^{r}$ then

$$
\text { Card WE }(g, G)= \begin{cases}0 & \text { if } r>2 g, \\ 1 & \text { if } r=1,2 g, \quad \text { or } 2 g-1 \\ k+1 & \text { if } 1<r<2 g-1\end{cases}
$$

In the rest of the paper we concentrate on the case when the genus $g$ of the surface is 2. Let $G=\bigoplus_{i=1}^{4} \mathbb{Z}_{r_{i}}$ with $r_{i}=p^{k_{i}}$ and $0 \leqq k_{1} \leqq k_{2} \leqq k_{3} \leqq k_{4}$. For simplicity, let $Y(n)$ be the $4 \times 4$ matrix whose diagonal is $\left(p^{k_{1}}, p^{k_{2}}, p^{k_{4}}, p^{k_{3}}\right)$ and the only other non-zero entry of which is $y_{34}=p^{n}$; let $Z(m, n ; w)$ be the $4 \times 4$ matrix whose diagonal is also $\left(p^{k_{1}}, p^{k_{2}}, p^{k_{4}}, p^{k_{3}}\right)$ but the other non-zero entries of which are $z_{32}=p^{m}$ and $z_{34}=w p^{n}$; and let $X(m)=Z(m, n ; 0)$. Here $(w, p)=1$. By Proposition 4.4 any matrix in $N(2, G)$ which is 
in a canonical form is equal to a $Z(m, n ; w)$ for some $m, n$ and $w$. In fact it is not difficult to see that:

4.7 Proposition. Every matrix in $N(2, G)$ is w-equivalent to one in one of the following forms:

(1) $X(m)$ with $k_{2} \leqq m \leqq \min \left\{k_{4}, k_{3}+k_{2}-k_{1}\right\}$,

(2) $Y(n)$ with $k_{3} \leqq n \leqq \min \left\{k_{4}, k_{3}+k_{2}-k_{1}\right\}$, or

(3) $Z(m, n ; w)$ such that

(i) $k_{3} \leqq n \leqq \min \left\{k_{4}, k_{3}+k_{2}-k_{1}\right\}$,

(ii) $n-k_{3}+k_{2}<m<n$,

(iii) $0<w<p \min \left\{k_{4}, k_{3}+m-k_{2}, k_{3}+k_{2}-k_{1}\right\}-n$, and

(iv) if $n=k_{3}$ then $w=1$.

Note that if $m=\min \left\{k_{4}, k_{3}+k_{2}-k_{1}\right\}$ then $X(m)$ is w-equivalent to the diagonal matrix $\operatorname{diag}\left(p^{k_{1}}, p^{k_{2}}, p^{k_{4}}, p^{k_{3}}\right)$.

4.8 Proposition.

(1) $X\left(m_{1}\right) \approx X\left(m_{2}\right)$ iff $m_{1}=m_{2}$.

(2) $Y\left(n_{1}\right) \approx Y\left(n_{2}\right)$ iff $n_{1}=n_{2}$.

(3) For each $m$ and $n, X(m) \neq Y(n)$.

(4) If $Z\left(m_{1}, n_{1} ; w_{1}\right) \approx Z\left(m_{2}, n_{2} ; w_{2}\right)$ then $m_{1}=m_{2}$ and $n_{1}=n_{2}$.

(5) For each $m_{1}, m_{2}, n$ and $w, X\left(m_{1}\right) \neq Z\left(m_{2}, n ; w\right)$.

(6) For each $m, n_{1}, n_{2}$ and $w, Y\left(n_{1}\right) \neq Z\left(m, n_{2} ; w\right)$.

Proof. (1): If $X\left(m_{1}\right)=S X\left(m_{2}\right) R$ for some $S$ in $S_{4}(\mathbb{Z})$ and $R$ in $G L_{4}(\mathbb{Z})$, then a comparison of the symplectic products of the first two columns of $X\left(m_{1}\right)$ and $X\left(m_{2}\right) R$ yields the equation:

$$
\left(r_{11} r_{22}-r_{12} r_{21}\right) p^{m_{2}}+\left(r_{11} r_{32}-r_{31} r_{12}\right) p^{k_{4}}+\left(r_{21} r_{42}-r_{22} r_{41}\right) p^{k_{3}+k_{2}-k_{1}}=p^{m_{1}},
$$

which has no integral solution unless $m_{1}=m_{2}$.

The proof of (2) is similar to that of (1).

(3): Suppose to the contrary that $X(m)=S Y(n) R$ for some $S$ in $S_{4}(\mathbb{Z})$ and $R$ in $G L_{4}(\mathbb{Z})$. The relations $J_{v}(X(m))=J_{S v}(Y(n))$ for $v$ in the set $\left\{\left(1,0,0, p^{m-k_{2}}\right),(0,1,0,0)\right.$, $(0,0,0,1)\}$ imply that either $p \mid \operatorname{det}(S)$ or

$$
\sum_{i=1}^{2} S(i, i+2 ; 1,2) \neq 0 \text {, }
$$

which contradicts the fact that $S$ is symplectic (see Lemma 4.2).

(4), (5), and (6): Similar arguments to those used in the proof of (1) imply that in these three cases one can assume that $m_{1}=m_{2}=m, m_{1}=m_{2}=m$, and $n_{1}=m$ respectively. Moreover, if $S$ in $\mathrm{Sp}_{4}(\mathbb{Z})$ and $R$ in $G L_{4}(\mathbb{Z})$ exist to make the relevant matrices wequivalent, then the $J_{v}()$ )-invariants for $v$ in the sets

$$
\begin{gathered}
\left\{\left(1, p^{m-k_{2}}, 0,-w p^{n_{1}-k_{3}}\right),(0,1,0,0),(0,0,0,1)\right\}, \\
\left\{\left(1,0,0, p^{m-k_{2}}\right),(0,1,0,0),(0,0,0,1)\right\},
\end{gathered}
$$


and

$$
\left\{\left(1,-p^{m-k_{3}}, 0,0\right),(0,1,0,0),(0,0,0,1)\right\}
$$

respectively imply that either $p \mid \operatorname{det}(S)$ or $\sum_{j=1}^{2} S(2,3 ; j, j+2) \neq 0$ in contradiction to the properties of the symplectic matrix $S$.

Unfortunately, we are unable to prove or disprove that $Z\left(m, n ; w_{1}\right) \approx Z\left(m, n ; w_{2}\right)$ iff $w_{1}=w_{2}$. However the previous two propositions can be used to enumerate the classes in $W E(2, G)$ for reasonably large class of groups and bounds can be given for the unsettled cases.

4.9 COROLlary. Let $K=\min \left\{k_{4}, k_{3}+k_{2}-k_{1}\right\}$.

(1) If $k_{2}=k_{3}$ or $k_{3}=k_{4}$ then $\operatorname{Card}(\operatorname{WE}(2, G))=K-k_{2}+1$.

(2) If $k_{2}=k_{1}+1, k_{3}=k_{2}+1$ or $k_{4}=k_{3}+1$ then $\operatorname{Card}(W E(2, G))=2\left(K-k_{2}\right)$.

(3) If $k_{i+1}>k_{i}+1$, for $i=1,2,3$, then

$$
1+\left(K-k_{2}\right)+\left(k_{3}-k_{2}\right)\left(K-k_{3}\right) \leqq \operatorname{Card}(W E(2, G)) \leqq 2\left(K-k_{2}\right)+L,
$$

where

and

$$
\begin{aligned}
L & =\sum_{i=1}^{r} \sum_{j=1}^{s}\left\{p^{f(i, j)}-1-(p-1)(f(i, j)-1)\right\}, \\
r & =K-k_{3}-1, \quad s=k_{3}-k_{2}-1,
\end{aligned}
$$

$$
f(i, j)=\min \left\{k_{4}-k_{3}-i, k_{2}-k_{1}-i, j\right\} \text {. }
$$

\section{REFERENCES}

1. J. S. Birman, On Siegel's modular group, Math. Ann. 191 (1971), 59-68.

2. A. L. Edmonds, Surface symmetry, Michigan Math. J. 29 (1982), 171-183.

3. B. Hartley and T. O. Hawkes, Rings modules and linear algebra (Chapman and Hall, 1970). 1980).

4. S. A. Jassim, Classifications of covering spaces (Ph.D. Thesis, University College of Swansea,

5. W. B. R. Lickorish, A finite set of generators for the homeotopy group of a 2-manifold, Proc. Cambridge Philos. Soc. 60 (1964), 769-778.

6. W. Magnus, A. Karass and B. Solitar, Combinatorial group theory, (John Wiley, 1966).

7. P. A. Smith, Abelian actions on 2-manifolds, Michigan Math. J. 14 (1967), 257-275.

11A Clarendon Road,

SKETTY,

Swansea SA2 OSR,

WALES 